\title{
Primitive small cell carcinoma of the prostate. Case report and revision of the literature
}

\author{
Pietro Pepe ${ }^{1}$, Ludovica Pepe ${ }^{1}$, Mara Curdman ${ }^{2}$, Michele Pennisi ${ }^{1}$, Filippo Fraggetta ${ }^{2}$ \\ ${ }^{1}$ Urology Unit - Cannizzaro Hospital, Catania, Italy; \\ ${ }^{2}$ Pathology Unit - Cannizzaro Hospital, Catania, Italy.
}

\begin{abstract}
Summary A Caucasian man 84 years old was admitted to our Department for acute renal failure secondary to severe bilateral hydronephrosis; moreover, the patient referred chronic fatigue and was anuric from two days. Serum creatinine and PSA values were equal to 9.6 $\mathrm{mg} / \mathrm{dl}$ and $4.8 \mathrm{ng} / \mathrm{ml}$ and digital rectal examination was highly suspicious for prostate cancer. In emergency, the patient underwent bilateral application of percutaneous renal nephrostomies to restore kidney function and, after three days, was submitted to ultrasound-guided extended transperineal biopsy; the histology showed the presence of a prostatic small cell carcinoma (SCC) fulfilling the World Health Organization criteria. The patient underwent clinical staging including chest and abdominal computed tomography evaluation and total body scan that did not demonstrated distant metastases and/or others primitive tumors; in addition, cystoscopy and urinary cytology were negative. The patient underwent multidisciplinary evaluation, but he died 20 days from the diagnosis for progressive clinical worsening (physical and cognitive impairments) before beginning oncological treatment.

In conclusion, primitive SCC represents a very rare cancer provided of poor prognosis; only the execution of prostate biopsy combined with an accurate specimen analysis allow to make the correct diagnosis and therapeutic treatment.
\end{abstract}

KEY WORDS: Prostate cancer; Small cell cancer; Prostate small cell cancer; Neuroendocrine small cell prostate cancer.

Submitted 17 July 2020; Accepted 30 September 2020

\section{INTRODUCTION}

Primitive small cell carcinoma (SCC) of the prostate is a very rare ( $0.5 \%$ of the cases) and aggressive type of prostatic cancer and often is diagnosed at advanced stage due to early metastasis (1-5); most frequently, SCC is associated to progressive hormone-refractory prostate cancer. The biology of SCC is poorly understood but it is always implicated in the lethal progression secondary to visceral metastases and lytic bones lesions. In this study, a case of primitive prostate SCC has been reported.

\section{Case report}

A Caucasian 84 years old man was admitted to our Department for acute renal failure secondary to severe bilateral hydronephrosis; the patient suffered from blood hypertension and was previously submitted to right inguinal hernia repair. The patient had not familiarity for prostate cancer ( $\mathrm{PCa}$ ) and assumed antihypertensive and alfa-blocker drugs. At admission, the patient referred chronic fatigue and was anuric from two days, serum creatinine and PSA values were equal to $9.6 \mathrm{mg} / \mathrm{dl}$ and $4.8 \mathrm{ng} / \mathrm{ml}$, respectively; moreover, digital rectal examination was highly suspicious for PCa. In emergency, the patient underwent bilateral application of percutaneous renal nephrostomies to restore kidney function. After three day the patient was submitted to ultrasound-guided extended transperineal biopsy (6); the histology showed the presence of a prostatic SCC fulfilling the World Health Organization criteria that involved all the 12 needle cores (Figure 1). The immunohistochemical analysis was positive for chromogranin, synaptophysin and focally for thyroid transcriptation factor 1 (TTF-1); Ki67 expression was equal to 85\% (Figure 2), conversely, CK7 and p63 were negative (7). The patient underwent clinical staging including chest and abdominal computed tomography (CT); evaluation and total body scan those did not demonstrated distant metastases and/or others primitive tumors; in addition, cystoscopy and urinary cytology were negative. The patient underwent multidisciplinary evaluation, but he died 20 days from the diagnosis for progressive clinical worsening (physical and cognitive impairments) before beginning oncological treatment. Autopsy was not performed.

\section{Discussion}

Primitive SCC of the prostate is a rare entity and has a presentation that is distinct from its adenocarcinoma counterparts (8); unique features include an unresponsiveness to hormonal therapy, rapid progression, increased risk of lytic bone lesions, presence of visceral metastases, and low PSA in relation to disease burden. On the other hand, the majority of prostate SCC are diagnosed in men with castration-resistant prostate cancer (CRPC) and are often characterized by the presence of neuroendocrine (NE) cancer differentiation.

The median time to development of SCC in patients with a history of prostatic adenocarcinoma is approximately 18-24 months from diagnosis, but ranges widely from few months to several years.

Primitive prostate SCC was first described by Wenk et al. (3) and, recently, Zhang et al. (4) reported 8 cases of pure SCC; autopsy studies of men who have died from CRPC 


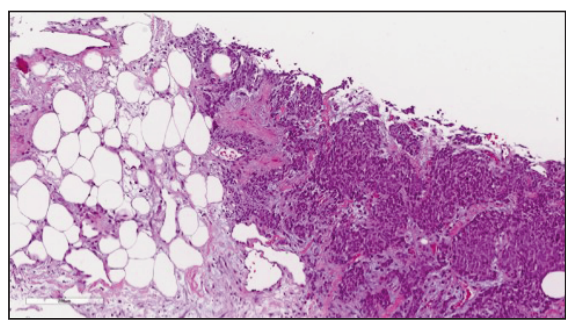

Figure 1.

Tumour was composed of undifferentiated cells with scant cytoplasm, infiltrating fatty tissue.

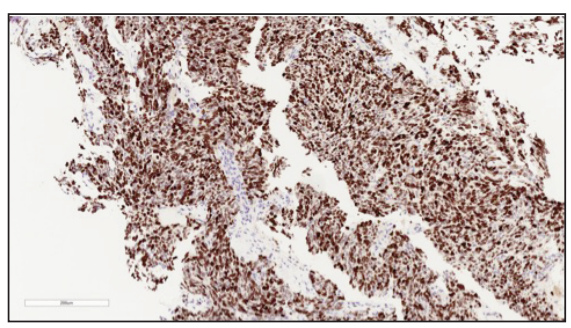

Figure 2.

Neoplastic cells showed a high proliferative fraction as highlighted by Ki67.

have reported the presence of SCC in up 10-20\% of cases. The histological diagnosis of SCC is reached based on the presence of morphological features similar to those found in SCC of the lung; using immunohistochemical techniques, the small-cell component could be positive for one or more neuroendocrine markers (i.e., neuron-specific enolase, synapthosiphysin, chromogranin, and CD56) in almost 90\% of the cases. Studies have demonstrated thyroid transcriptation factor 1 (TYF-1) expression in over $50 \%$ of SCCs of the prostate, $85-90 \%$ loss rate of tumor suppressor retinoblastoma protein (RB1), and 50-60\% mutation rate of TP53, limiting its utility in distinguishing primary prostate SCC from metastases of other small cell cancers. Therefore, in the absence of a primary SCC at another site (for example, the lung), the finding of SCC on prostate biopsy is almost undoubtedly an indication of prostatic origin.

In the last years, gene expression in metastatic CRPC neuroendocrine SCC prostate has been tested confirming similar molecular profile with small cell lung carcinoma (8). In addition, molecular studies have reported an increased expression of gene involved in cellular proliferation, cell cycle, neuroendocrine differentiation and mitosis.

Metzger et al. (5) reported on 657 men with neuroendocrine SCC an overall survival of 12 months. Wang et al. (9) on 260 patients selected from Surveillance, Epidemiology, and End Results (SEER) database showed an increased incidence of prostate SCC over time that was characterized by presence of high stage (stage IV in $77.7 \%$ ), nodes involvement (49\%) and distant metastases (68\%) with PSA values greater than $10 \mathrm{ng} / \mathrm{ml}$ only in $23 \%$ of the cases; although patients underwent chemotherapy (58.8\%), surgery (25.4\%) and, radiotherapy (31.9\%) the observed survival rates of 1-year, 2-year, and 5-year were $42.1 \%, 22.1 \%$, and $12.5 \%$, respectively.

Although the poor prognosis, it is important to make distinction between primitive prostate SCC and NE-CRPC because therapeutic strategy changes and should be managed similarly to other non-prostatic SCC. Rarely prostatectomy or radiotherapy alone have been shown to be curative because the tumor, in the majority of the cases, is metastatic (5), moreover, prostate SCC is usually not responsive to androgen deprivation and disease progression is not associated with rises in serum PSA values. In definitive, the best treatment remains the multidisciplinary approach by chemotherapy alone or combined with local therapy that could offer local palliation of clinical symptoms $(5,10)$.

In our case, the patient had a primitive prostate SCC characterized as locally advanced cancer involving bladder and kidney function in absence of documented distant metastases; therefore, we don't know if the patient died from prostate SCC or from concomitant onset systemic pathologies.

In conclusion, although prostate SCC could be suspected in men with CRPC with clinical progression, primitive SCC represents a very rare cancer provided of poor prognosis and only prostate biopsy combined with an accurate specimen analysis allow to make the correct diagnosis and therapeutic treatment.

\section{REFERENCES}

1. Terada T. Small cell neuroendocrine carcinoma of the prostate: incidence and a report of four cases with an examination of KIT and PDGFRA. Prostate. 2012; 72:1150-1156.

2. Wang W, Epstein JI. Small cell carcinoma of the prostate. A morphologic and immunohistocemical study of 95 cases. Am J Surg Pathol. 2008; 32:65-71.

3. Wenk RE, Bhagavan BS, Levy R, et al. Ectopic ACTH, prostatic oat cell carcinoma, and marked hypernatremia. Cancer. 1977; 40:773-778

4. Zhang Y, Ouyang W, Sun G, et al. Pure small cell carcinoma of prostate: A report of 8 cases. Urol Int. 2018; 101:263-268.

5. Metzger AL, Abel S, Wegner RE, et al. Patterns of care and outcomes in small cell carcinoma of the prostate: A national cancer database analysis. Prostate 2019; 79:1457-1461.

6. Pepe P, Garufi A, Priolo GD, et al. Is it time to perform only magnetic resonance imaging targeted cores? Our experience with 1,032 men who underwent prostate biopsy. J Urol. 2018; 200:774-778.

7. Pepe P, Fraggetta F, Candiano G, Aragona F. Does Ki-67 staining improve quantitative histology in preoperative prostate cancer staging? Arch Ital Urol Androl. 2012; 84:32-35.

8. Salemi M, Galia A, Fraggetta F, et al. Poly (ADP-ribose) polymerase 1 protein expression in normal and neoplastic prostatic tissue. Eur J Histochem. 2013; 57:e13.

9. Wang J, Liu X, Wang Y, Ren G. Current trend of worsening prognosis of prostate small cell carcinoma: A population-based study. Cancer Med. 2019; 8: 6799-6806.

10. Papandreou CN, Daliani DD, Thall PF, et al. Results of a phase II study with doxorubicin, etoposide, and cisplatin in patients with fully characterized small-cell carcinoma of the prostate. J Clin Oncol. 2002; 20:3072-3080.

\section{Correspondence}

Pietro Pepe, MD (Corresponding Author)

piepepe@hotmail.com

Michele Pennisi, MD

Ludovica Pepe

Urology Unit - Cannizzaro Hospital, Catania

Via Messina 829, Catania (Italy)

Mara Curdman, MD

Filippo Fraggetta, MD

Pathology Unit - Cannizzaro Hospital, Catania (Italy) 